\title{
Deporte alternativo: una mirada sociológica ejemplificada desde el tchoukball ${ }^{*}$
}

\author{
Andrés Fabián Carrillo Brugos** \\ Orlando Granados López*** \\ Samuel Jiménez Gómez ${ }^{* * * *}$
}

Recibido: octubre 8 de 2016 • Evaluado: octubre 19 de 2016

Aceptado: octubre 28 de 2016

\section{Resumen}

El deporte alternativo: una mirada sociológica ejemplificada desde Tchoukball es una investigación académica que busca localizar los aspectos sociológicos más importantes de los deportes alternativos, tomando como ejemplo el tchoukball. En cuanto a la recolección de la información, se eligen dos poblaciones de estudio, un grupo con el que se llevó a cabo una entrevista; al segundo grupo de estudio, se le aplicó una encuesta. Los resultados que aquí se exponen, se presentan en unas categorías de análisis cualitativo. Finalmente, la investigación que se realiza está apoyada por una revisión de autores, tanto nacionales como internacionales; con ello se intenta proponer un enfoque de opinión con base en el contexto colombiano.

Palabras clave: deporte alternativo, sociología, deporte, Tchoukball.

" El presente artículo deriva de un proceso de formación en investigación en el espacio académico Sociología del deporte, realizado durante el periodo 2016.

"* Estudiante Facultad de Cultura Física, Deporte y Recreación.

"** Estudiante Facultad de Cultura Física, Deporte y Recreación.

"**** Estudiante Facultad de Cultura Física, Deporte y Recreación. 


\section{Alternative sport: a sociological look exemplified from tchoukball}

\section{Abstract}

Alternative sport: a sociological view exemplified by Tchoukball, is an academic research that seeks to locate the most important sociological aspects of alternative sports, taking Tchoukball as an example. As for the collection of information, two study populations are chosen. In the first group, the an interview was made. For the case of the second study group, the interview format was used for five relatives of the young people interviewed, who do not formally practice this sport. The results shown here are presented in categories of analysis (qualitative). Finally, the research carried out is supported by a review of authors, both national and international, and with this we try to propose an opinion approach based on the Colombian context.

Keywords: Alternative Sport, Sociology, Sport, Tchoukball. 


\section{Introducción}

El deporte alternativo nace en la década de los 70 como deporte y deja de ser considerado como un simple juego. Bajo este marco se buscaba la oposición hacia los modelos económicos modernos de los deportes convencionales. Sobre esto, Sancho (2013) menciona que la categoría de "deportes alternativos se produjo en España en la década de los ochenta. Los juegos y deportes alternativos fueron integrados en el currículo de Educación Física como reacción al modelo deportivo imperante, basado en el rendimiento" (p. 1).

Según Verdú (2008), el deporte alternativo es un "medio para la introducción de nuevos hábitos deportivos, dando mayor importancia a los aspectos recreativos de relación, cooperación y comunicación que a los aspectos relacionados con la competición" (p. 88). Posteriormente, se identifica que a pesar de que estas prácticas deportivas están siendo implementadas en entornos escolares y que algunas de sus disciplinas gozan de gran reconocimiento entre la población en general (patinaje, skate, paracaidismo), esta clasificación del deporte no tiene un concepto homogéneo y unificado. Respecto a este aspecto, un concepto adecuado que define el deporte alternativo es el de Deporte Alternativo (2010) que plantea que "son aquellos que no se hallan sujetos a los círculos tradicionales de fabricación para el campo de las actividades físicas deportivas o recreativas" (p. 1). Por otra parte, Rojas (2008) citando a Feixa (1995) se refiere a los deportes alternativos "como un campo en el que se mezclan tres visiones de la vida y del mundo: la visión física externa, naturaleza, agua, velocidad; la visión emotiva interna, riesgo, libertad y la visión química resultante, adrenalina” (p. 15).

Es importante destacar que los deportes alternativos incluyen nuevos hábitos deportivos en la sociedad, puesto que se presta mayor atención a las relaciones de recreación, cooperación y comunicación entre sus participantes; dejando en un segundo plano la relación de la competición, como sucede en los deportes convencionales.

Desde el contexto local (Bogotá, Colombia), a partir del año 2012, existe el programa de Deportes Urbanos y Nuevas Tendencias (DUNT), el cual se ocupa de otorgar cursos y escenarios específicos de los diferentes deportes alternativos en la ciudad de Bogotá; esto con el fin de erradicar problemáticas como lo son las pandillas o la drogadicción y fomentar el uso 
adecuado del tiempo libre. En sus inicios el programa contaba con 14 modalidades deportivas: skate; roller; BMX en las modalidades park, flatland, dirt jump y street; longboarding; roller derby; parkour; capoeira; bike polo; y freestyle fútbol. Sin embargo, tras el paso de los años, se han añadido otras tendencias como el tchoukball y el slackline.

Los escenarios específicos de los deportes urbanos están distribuidos en los principales parques de la ciudad, específicamente 8 de estos. También es importante señalar que este programa cuenta con la participación de más de 25.000 practicantes de diferentes tendencias deportivas $\mathrm{y}$, de igual forma, miembros de diferentes comunidades urbanas. A pesar de que estas tendencias deportivas no poseen un reconocimiento tan amplio como los demás deportes convencionales, sí existe una intención real de muchas personas que se esfuerzan por apoyar a los mejores talentos en competiciones de gran reconocimiento social, tanto a nivel nacional como internacional. Suárez (2013) indica que:

La meta del programa DUNT es conformar la Selección Bogotá de Deportistas Urbanos que represente a nivel nacional e internacional a la ciudad con el apoyo metodológico que tienen los deportes tradicionales para llevarlo a lo más alto de los podios. (p. 1)

En cuanto al tchoukball, es un deporte que nace en 1970 en Suiza, creado por el biólogo Dr. Hermann Brandt. Según Barnechea (2010), citando a Brandt, expresa que "el objetivo de las actividades físicas no es fabricar campeones, sino contribuir a la edificación de una sociedad armoniosa” (p. 13). Este deporte es el único que ha recibido el premio Thulin, por contribuir al desarrollo de la educación física y también es avalado por la UNESCO como el deporte de la paz. Este deporte excluye toda búsqueda de prestigio puesto que Barnechea (2010) dice que

En el plano colectivo, el resultado cualquiera que sea no compromete la reputación de nadie y, sobretodo, no da lugar a ninguna clase de "sectarismo". Con una victoria se puede sentir placer o alegría, pero nunca satisfacción de orgullo. La alegría de ganar es un estímulo. El orgullo de la victoria comporta un germen de lucha de prestigio que nosotros condenamos como fuente de conflictos inter humanos a todos los niveles (p. 15). 
En la zona 11 de la ciudad de Bogotá, en el club deportivo Linces 78 , se puede apreciar la práctica de este deporte, donde la mayoría de sus practicantes están entre las edades de 8 a 19 años. Con el propósito de identificar y analizar los aspectos sociológicos de los deportes alternativos, se va a tener en cuenta los datos recopilados, tanto de los practicantes de esta disciplina deportiva como de los no practicantes. A patir de lo anterior, el presente ejecicio investigativo buscó identificar los aspectos sociológicos que se presentan en los deportes alternativos.

Finalmente, y desde el punto de vista de la sociología y el enfoque que se proyecta para la investigación, se destaca el concepto de la inclusión y su importancia. En el 2007, Molina infiere que se deben crear espacios que se consideren convenientes para las personas, fomentando estrategias y herramientas de aprendizaje colaborativas, todo esto para contribuir al desarrollo integral de los individuos.

Según la RAE (s.f) la inclusión se define como: "Conjunto de rasgos propios de un individuo o de una colectividad que los caracterizan frente a los demás". Por lo anterior, McLaren y Tofler señalan que

Los jóvenes tienen dificultades para configurar su identidad en las instituciones tradicionales como la familia, la iglesia y la escuela, estos encuentran unos nuevos referentes producidos por la industria cultural a los que se adscriben como forma de identidad y que aparentemente son más atractivos, ya que hacen lectura de lo que es ser joven actualmente (citados por Rojas, 2008, p. 7).

\section{Metodología}

La población de estudio está divida en dos grupos. El primero estuvo conformado por cinco practicantes de Tchoukball que están entre las edades de 8 y 19 años; todos ellos son representantes de Colombia en los diferentes torneos internacionales -en este mismo grupo se incluye al entrenador del equipo-. El segundo grupo está conformado por cinco padres de los practicantes del primer grupo, entre los 30 y los 60 años.

Los instrumentos de recolección de información, utilizados fueron: 
- Entrevista: Se compone de cinco preguntas abiertas, las cuales se realizaron tanto a los cinco practicantes del Tchoukball, como también al entrenador del equipo.

- Encuesta: Conformada por nueve preguntas: una de selección múltiple y única respuesta, siete de selección múltiple con única respuesta y complementada con argumentos personales. Por último, se presenta una pregunta de selección múltiple, con más de una respuesta.

\section{Objetivos}

- Identificar los aspectos sociales más representativos del deporte alternativo, más exactamente en el Tchoukball

- Establecer cuáles son las diferencias entre el deporte alternativo y el deporte convencional

- Analizar los diferentes estratos socioeconómicos que predominan en la práctica del Tchoukball

\section{Resultados}

En primer lugar, se exponen los resultados de las entrevistas realizadas a los cinco practicantes de tchoukball, junto con la participación de su correspondiente entrenador. En segundo lugar, se presentan los resultados de las encuestas realizadas al segundo grupo de investigación, el cual está conformado por los cinco padres de los atletas del primer grupo de estudio. Para identificar los resultados de los dos grupos se utilizaron las siguientes convenciones:

1. Grupo de estudio \#1: Conformado por cinco deportistas y un entrenador. Las convenciones de cada uno de estos se establecen de acuerdo al orden de las preguntas formuladas y su respectiva intervención:

$$
\begin{aligned}
& \text { 1er Deportista }=\text { D1 } \\
& \text { 2do Deportista }=\text { D2 } \\
& \text { 3er Deportista }=\text { D3 } \\
& \text { 4to Deportista }=\text { D4 } \\
& \text { 5toDeportista = D5 } \\
& \text { Entrenador = E }
\end{aligned}
$$


2. Grupo de estudio \#2: Conformado por cinco padres de familia. Para este caso, las convenciones se estructuran de la siguiente forma:

1era Acudiente $=\mathrm{A} 1$

2 da Acudiente $=\mathrm{A} 2$

3 era Acudiente $=\mathrm{A} 3$

4 to Acudiente $=$ A4

5 to Acudiente $=\mathrm{A} 5$

En las siguientes tablas, se reconocen los resultados específicos de los dos instrumentos de recolección de la información, organizados de acuerdo con las categorías de análisis:

\begin{tabular}{|c|c|c|c|}
\hline \multicolumn{4}{|c|}{ RESULTADOS - ENTREVISTAS } \\
\hline \multirow{2}{*}{$\begin{array}{l}\text { Pregunta } \\
\text { de } \\
\text { encuesta }\end{array}$} & \multicolumn{3}{|c|}{ Categorías de análisis } \\
\hline & Convergente & Divergente & Emergente \\
\hline 1 & $\begin{array}{l}\text { D1 y D2 concuer- } \\
\text { dan en que, un de- } \\
\text { porte alternativo, es } \\
\text { un deporte diferente } \\
\text { a los convencionales } \\
\text { y que hace que sea } \\
\text { diferente la forma } \\
\text { de vivir el deporte. }\end{array}$ & $\begin{array}{l}\text { D3 Indica que los } \\
\text { deportes alterna- } \\
\text { tivos son todos } \\
\text { nuevos dentro del } \\
\text { contexto mundial. } \\
\text { También hubo otra } \\
\text { respuesta diferen- } \\
\text { te por parte del } \\
\text { individuo E en la } \\
\text { cual afirma que el } \\
\text { deporte alternativo } \\
\text { es una propuesta } \\
\text { diferente expuesta } \\
\text { a todo el mundo } \\
\text { para la realización } \\
\text { de alguna actividad } \\
\text { física. }\end{array}$ & $\begin{array}{l}\text { El participante } \\
\text { D4 infiere que los } \\
\text { deportes alterna- } \\
\text { tivos buscan en } \\
\text { algún momento ser } \\
\text { considerados como } \\
\text { convencionales. }\end{array}$ \\
\hline
\end{tabular}




\begin{tabular}{|c|c|c|c|}
\hline \multicolumn{4}{|c|}{ RESULTADOS - ENTREVISTAS } \\
\hline \multirow{2}{*}{$\begin{array}{l}\text { Pregunta } \\
\text { de } \\
\text { encuesta }\end{array}$} & \multicolumn{3}{|c|}{ Categorías de análisis } \\
\hline & Convergente & Divergente & Emergente \\
\hline 3 & & $\begin{array}{l}\text { D1 menciona que el } \\
\text { tchoukball ha gene- } \\
\text { rado un montón de } \\
\text { sentimientos, adqui- } \\
\text { riendo responsabi- } \\
\text { lidades, autonomía, } \\
\text { invitando a vivir } \\
\text { de otra manera el } \\
\text { deporte, por otra } \\
\text { parte, D2 considera } \\
\text { que los sentimientos } \\
\text { que generan son } \\
\text { más de compartir } \\
\text { y de conocer otros } \\
\text { tipos de culturas y } \\
\text { otras maneras de } \\
\text { concebir el depor- } \\
\text { te y finalmente el } \\
\text { deportista E indica } \\
\text { que el tchoukball } \\
\text { puede llegar a } \\
\text { generar amistad, } \\
\text { fraternidad, alegría } \\
\text { y unión provocan- } \\
\text { do un trabajo en } \\
\text { grupo. }\end{array}$ & $\begin{array}{l}\text { D5 dice que te sien- } \\
\text { tes diferente a los } \\
\text { demás solo por el } \\
\text { hecho de practicar } \\
\text { tchoukball, favore- } \\
\text { ciendo el desarrollo } \\
\text { intrapersonal. }\end{array}$ \\
\hline 4 & $\begin{array}{l}\text { D5 y D2 dicen que } \\
\text { predominan los } \\
\text { estratos de clase } \\
\text { media, aunque tam- } \\
\text { bién dicen que no } \\
\text { es un deporte que } \\
\text { se rige por valores } \\
\text { económicos y el } \\
\text { practícate E y D1 } \\
\text { concuerdan en que } \\
\text { es un deporte para } \\
\text { cualquier estrato so- } \\
\text { cial, debido a que es } \\
\text { un deporte equitati- } \\
\text { vo en la forma que } \\
\text { se integran las clases } \\
\text { socioeconómicas. }\end{array}$ & $\begin{array}{l}\text { El participante D4 } \\
\text { hace la distinción } \\
\text { que, debido a ser el } \\
\text { deporte de la paz, } \\
\text { está desarrollado } \\
\text { para que todos lo } \\
\text { jueguen. }\end{array}$ & $\begin{array}{l}\text { D3 de los partici- } \\
\text { pantes menciona } \\
\text { que debido a su } \\
\text { flexibilidad en el } \\
\text { reglamento y en sus } \\
\text { instrumentos con } \\
\text { que se desarrolla, } \\
\text { se puede jugar por } \\
\text { cualquier estrato } \\
\text { social, y que solo } \\
\text { importa el amor por } \\
\text { el tchoukball. }\end{array}$ \\
\hline
\end{tabular}




\section{RESULTADOS - ENTREVISTAS}

\begin{tabular}{|c|c|c|c|}
\hline \multirow{2}{*}{$\begin{array}{l}\text { Pregunta } \\
\text { de } \\
\text { encuesta }\end{array}$} & \multicolumn{3}{|c|}{ Categorías de análisis } \\
\hline & Convergente & Divergente & Emergente \\
\hline 5 & $\begin{array}{l}\text { Participantes } \\
\text { D3 y D2 dicen } \\
\text { que, debido a su } \\
\text { característica del } \\
\text { normal desarrollo } \\
\text { de la práctica, } \\
\text { en donde no hay } \\
\text { contacto físico, } \\
\text { los jugadores se } \\
\text { dedican a jugar y } \\
\text { no a buscar por } \\
\text { cualquier medio } \\
\text { solo ganar, caso que } \\
\text { ocurre en el deporte } \\
\text { convencional. }\end{array}$ & $\begin{array}{l}\text { El participante } \\
\text { D4 afirma que } \\
\text { la finalidad del } \\
\text { tchoukball es } \\
\text { mejorar, aprender, } \\
\text { enseñar y no } \\
\text { ganar, en cambio } \\
\text { el deporte } \\
\text { convencional solo } \\
\text { se preocupa por el } \\
\text { resultado final } \\
\text { El entrevistado } \\
\text { E indica que el } \\
\text { tchoukball se } \\
\text { presenta como } \\
\text { una propuesta } \\
\text { deportiva diferente } \\
\text { que involucra } \\
\text { varios deportes } \\
\text { convencionales }\end{array}$ & $\begin{array}{l}\text { El individuo D1 } \\
\text { indica que en el } \\
\text { trascurso de la } \\
\text { práctica de este } \\
\text { deporte si alguien } \\
\text { del equipo contrario } \\
\text { no está haciendo las } \\
\text { cosas de la mejorar } \\
\text { manera, se le dará } \\
\text { ánimo y consejos } \\
\text { para que este pueda } \\
\text { desarrollar con } \\
\text { plenitud la misión u } \\
\text { objetivo establecido } \\
\text { del juego. }\end{array}$ \\
\hline
\end{tabular}




\section{RESULTADOS - ENTREVISTAS}

\begin{tabular}{|c|c|c|c|}
\hline \multirow{2}{*}{$\begin{array}{c}\text { Pregunta } \\
\text { de encuesta }\end{array}$} & \multicolumn{3}{|c|}{ Categorías de análisis } \\
\hline & Convergente & Divergente & Emergente \\
\hline 1 & $\begin{array}{l}\text { A1, A2, A3 Y } \\
\text { A5, marcaron } \\
\text { la opción "Sí", } \\
\text { contestando de } \\
\text { manera correcta } \\
\text { que sí cono- } \\
\text { cen el deporte } \\
\text { alternativo. }\end{array}$ & & $\begin{array}{l}\text { A4 marco la } \\
\text { opción "No"; } \\
\text { en donde se } \\
\text { evidencia que } \\
\text { no sabe que } \\
\text { es un deporte } \\
\text { alternativo. }\end{array}$ \\
\hline 2 & $\begin{array}{l}\text { A1, A2 y A5 } \\
\text { mencionaron al } \\
\text { menos un deporte } \\
\text { alternativo. }\end{array}$ & $\begin{array}{l}\text { A3 no mencionó } \\
\text { ningún } \\
\text { ejemplo, pero } \\
\text { sí reconoce sus } \\
\text { características. }\end{array}$ & $\begin{array}{l}\text { A4 no contesto } \\
\text { a esta pregunta, } \\
\text { debido a que } \\
\text { no sabe que } \\
\text { es un deporte } \\
\text { alternativo. }\end{array}$ \\
\hline 3 & $\begin{array}{l}\text { Todos los } \\
\text { encuestados, } \\
\text { contestaron que } \\
\text { no practica y } \\
\text { tampoco han } \\
\text { practicado un de- } \\
\text { porte alternativo. }\end{array}$ & & \\
\hline 4 & $\begin{array}{l}\text { Todos los encues- } \\
\text { tados concuerdan } \\
\text { en que no reco- } \\
\text { nocen a algún de- } \\
\text { portista nacional } \\
\text { o internacional } \\
\text { famoso dedicado } \\
\text { profesionalmen- } \\
\text { te a la práctica } \\
\text { de los deportes } \\
\text { alternativos. }\end{array}$ & & \\
\hline
\end{tabular}


RESULTADOS - ENTREVISTAS

\begin{tabular}{|c|c|c|c|}
\hline \multirow{2}{*}{$\begin{array}{l}\text { Pregunta } \\
\text { de encuesta }\end{array}$} & \multicolumn{3}{|c|}{ Categorías de análisis } \\
\hline & Convergente & Divergente & Emergente \\
\hline 5 & $\begin{array}{l}\text { Todos los encues- } \\
\text { tados contestaron } \\
\text { que sí están de } \\
\text { acuerdo de que } \\
\text { su hijo(a) siga } \\
\text { practicando el de- } \\
\text { porte alternativo. }\end{array}$ & $\begin{array}{l}\text { En cuanto a } \\
\text { sus razones, se } \\
\text { puede identifi- } \\
\text { car que A3, A4 } \\
\text { y A5 coinciden } \\
\text { en mencionar el } \\
\text { bienestar de sus } \\
\text { hijos ya sea me- } \\
\text { diante la práctica } \\
\text { del deporte alter- } \\
\text { nativo, como del } \\
\text { deporte conven- } \\
\text { cional; mientras } \\
\text { que A2 indica } \\
\text { que los depor- } \\
\text { tes alternativos } \\
\text { mejoran sus ca- } \\
\text { pacidades físicas. } \\
\text { Por último, la } \\
\text { encuestada A1 no } \\
\text { señala ninguna } \\
\text { razón. }\end{array}$ & $\begin{array}{l}\text { A3 contestó una } \\
\text { opción adicional, } \\
\text { indicando que sí } \\
\text { estaba de acuerdo } \\
\text { de que su hija, } \\
\text { también conti- } \\
\text { nuara practican- } \\
\text { do los deportes } \\
\text { convencionales. }\end{array}$ \\
\hline 6 & & $\begin{array}{l}\text { Los encuestados } \\
\text { A1, A2, A3 y } \\
\text { A5 contestan } \\
\text { algunas opciones } \\
\text { de respuesta que } \\
\text { son válidas, a } \\
\text { pesar de que sus } \\
\text { respuestas son } \\
\text { diferentes, al con- } \\
\text { testar una opción } \\
\text { adicional u omitir } \\
\text { alguna. }\end{array}$ & $\begin{array}{l}\text { A4 contestó la } \\
\text { opción: "No Sabe } \\
\text { / No responde". }\end{array}$ \\
\hline
\end{tabular}




\begin{tabular}{|c|c|c|c|}
\hline \multicolumn{4}{|c|}{ RESULTADOS - ENTREVISTAS } \\
\hline \multirow{2}{*}{$\begin{array}{l}\text { Pregunta } \\
\text { de encuesta }\end{array}$} & \multicolumn{3}{|c|}{ Categorías de análisis } \\
\hline & Convergente & Divergente & Emergente \\
\hline 7 & $\begin{array}{l}\text { Todos los } \\
\text { encuestados, in- } \\
\text { dican que no han } \\
\text { tenido ninguna } \\
\text { experiencia nega- } \\
\text { tiva en la práctica } \\
\text { de algún deporte } \\
\text { alternativo. }\end{array}$ & & \\
\hline 8 & & $\begin{array}{l}\text { Los encuestados } \\
\text { A1 y A5, señalan } \\
\text { la opción: No } \\
\text { sabe. Por otra } \\
\text { parte, A2 elije } \\
\text { la opción "No", } \\
\text { aunque sus razo- } \\
\text { nes no sean las } \\
\text { más convenientes } \\
\text { a la pregun- } \\
\text { ta planteada. } \\
\text { Por último, los } \\
\text { encuestados A3 } \\
\text { y A4 indican la } \\
\text { opción "Sí" y } \\
\text { concuerdan que } \\
\text { los elementos } \\
\text { para llevar a cabo } \\
\text { la práctica de un } \\
\text { deporte alternati- } \\
\text { vo influyen en la } \\
\text { persona al decidir } \\
\text { practicarlo. }\end{array}$ & \\
\hline
\end{tabular}




\begin{tabular}{|c|c|c|c|}
\hline \multicolumn{4}{|c|}{ RESULTADOS - ENTREVISTAS } \\
\hline \multirow{2}{*}{$\begin{array}{l}\text { Pregunta } \\
\text { de encuesta }\end{array}$} & \multicolumn{3}{|c|}{ Categorías de análisis } \\
\hline & Convergente & Divergente & Emergente \\
\hline 9 & & $\begin{array}{l}\text { Las encuestadas } \\
\text { A2 y A3 coinci- } \\
\text { den en que las } \\
\text { razones por las } \\
\text { cuales el deporte } \\
\text { alternativo no tie- } \\
\text { ne tanta atención } \\
\text { por parte de los } \\
\text { MC. Se debe al } \\
\text { reconocimiento } \\
\text { o popularidad de } \\
\text { estos deportes y } \\
\text { también por falta } \\
\text { de publicidad. } \\
\text { Mientras que } \\
\text { para A5, propone } \\
\text { que el marketing } \\
\text { es superior en } \\
\text { los deportes que } \\
\text { más venden (se } \\
\text { presume que los } \\
\text { convencionales) } \\
\text { y por ello, las in- } \\
\text { dustrias obtienen } \\
\text { más beneficios. } \\
\text { Por último, A4 } \\
\text { no contesta la } \\
\text { pregunta. }\end{array}$ & $\begin{array}{l}\text { A1 realiza } \\
\text { una distinción } \\
\text { entre medios de } \\
\text { comunicación } \\
\text { convencionales y } \\
\text { alternativos; en el } \\
\text { primero plantea } \\
\text { que solamente se } \\
\text { preocupan por el } \\
\text { Rating; y en el se- } \\
\text { gundo considera } \\
\text { que sí buscan este } \\
\text { tipo de deporte } \\
\text { para presentarlo } \\
\text { ante el público. }\end{array}$ \\
\hline
\end{tabular}




\section{Discusión}

Según los resultados, se encuentran similitudes entre lo que dicen otros autores y los resultados que aparecen en la investigación. Barnechea (2010) indica que el deporte alternativo está asociado a actividades cooperativas, lúdicas, recreativas o competitivas las cuales son innovadoras. Por lo anterior, los investigadores distinguen la forma en la que se vive el deporte a partir de las dos poblaciones de estudio que fueron consultadas en la aplicación de los instrumentos de recolección, en estas se destaca la diferencia entre el deporte alternativo y el deporte convencional; un ejemplo de ello, es que el foco de atención del deporte alternativo, se centra en el crecimiento personal e integral de los individuos, mientras que el deporte convencional o tradicional se centra en el crecimiento de los individuos, entorno a las capacidades físicas de los mismos.

Otro aspecto importante es que, el deporte convencional o tradicional, en el que Isoma et al. (2014) concluyen en su estudio cuando plantean que "los chicos adolescentes practican más deporte que las chicas, especialmente cuando se trata de deporte federado" (p. 84). De esta manera en los resultados se destaca el aspecto social de la integración entre mujeres y hombres en la práctica del deporte alternativo, debido a que este tipo de deportes favorece la misma dedicación de tiempo por parte de los dos géneros.

Por otra parte, desde el aspecto de la inclusión, Raudsepp y Viira (2000) indican que los hombres eligen realizar sus actividades deportivas de forma colectiva, mientras que las mujeres, prefieren realizarla de forma individual. Sin embargo, los resultados revelan que los deportes alternativos y específicamente el tchoukball, generan espacios de inclusión donde estos dos géneros se encuentran en las mismas posibilidades e igualdad en la práctica.

A manera de conclusión, se identifican cinco aspectos sociológicos que se presentan en los deportes alternativos, los cuales son: diversión, participación social, la integración e inclusión y mentalidad que se tiene en los partidos. Este último es un aspecto que complementa la temática presentada, además de ser una novedad en la investigación; es explicado por parte de uno de los practicantes del Tchoukball, al proponer que no hay una rivalidad conflictiva donde solo se busca el bien para sí mismo. En esta mentalidad alternativa, él afirma que, a pesar de existir una distinción de equipos, se busca el bien colectivo, el cual se constituye a partir de las relaciones de 
"mejorar, aprender y enseñar" y no solamente en ganar el partido. Por otra parte, sorprende destacar que, uno de los encuestados desconoce la identidad del deporte alternativo al confundirlo con un deporte convencional. Eso demuestra que existe un apoyo por parte del acudiente, pero no hay una aprobación personal y sí hay un desinterés por la práctica que realiza su hijo.

A manera de propuesta, los investigadores en formación invitan a la práctica de este tipo de deportes, debido a sus características lúdicas y a la forma en que generan entornos de esparcimiento en diferentes grupos poblacionales. Otro aspecto importante, es que las instituciones educativas (escolares y universitarias) deberían incluir en sus currículos este tipo de disciplinas deportivas, ya que estas proporcionan a estos contextos educativos las alternativas del deporte que fortalecen sus competencias personales y cívicas, que no logran complementar desde la práctica del deporte convencional.

\section{Referencias}

Barnechea, C. (2010). El tchoukball: Un deporte alternativo para todos. Sevilla: Wanceulen SL.

Feu Molina, S. (2007). Los juegos y deportes alternativos: entre la utopía y la posibilidad de la coeducación en la educación física. VIII Congreso de Estudios Extremeños. 1676-1692.

Fuster, J., Funollet, F. \& Gómez, J. (2008). Características elementales de los nuevos deportes en el medio natural. Ágora para la educación física y el deporte, 7 (8) 35 - 47. Recuperado de https:/dialnet.unirioja.es/servlet/ articulo?codigo $=2725426$

García, A. (2013) Trabajo de fin de grado: los deportes alternativos en el deporte escolar. (Tesis de posgrado). Universidad de Valladolid. Valladolid, España.

García, J. C (2013, 11 de noviembre) Bogotá reclama su espacio para el deporte urbano. Ideal.es. Recuperado de http://granadablogs.com/gr-arquitectos/2013/11/11/ bogota-reclama-su-espacio-para-el-deporte-urbano/

Isoma, M., Boubeta, A. \& Vaquero, R. (2014). Motivaciones para la práctica deportiva en escolares federados y no federados. Retos - Nuevas tendencias de en Educación Física, Deporte y Recreación, 1 (25), 80 - 84. Recuperado de https://dialnet.unirioja.es/servlet/articulo?codigo $=4554982$ 
Rojas, R (2008) El deporte extremo urbano como parte de la Cultura Juvenil, (Monografía de Pregrado). Universidad Santo Tomás. Bogotá, CO.

Sancho, J (2013) Juegos y deportes alternativos. Educación Física en la nube. Recuperado de: http://efedublog.blogspot.com.co/2013/10/juegos-y-deportesalternativos.html

Sandra Dora, E (2013). Deportes Alternativos: Una propuesta actualizada para abordar la perspectiva de género. Revista de formación $n^{\circ} 1$. 10mo Congreso Argentino de Educación Física y Ciencias.

Splashy Templates (2010) Deporte alternativo. Bogotá, C. Deportes alternativos blog spot. Recuperado de: http://deportesmasalternativos.blogspot.com.co

Suárez, J (2013, 25 de enero) Deporte Urbanos: 25 mil practicantes en Bogotá. El Nuevo siglo.com. Recuperado de http://www.elnuevosiglo.com.co/ articulos/1-2013-deportes-urbanos-25-mil-practicantes-en-bogota

Thorpe, H. (2009). Understanding 'Alternative' sport Experiences: a contextual Approach for sport Psychology. The International Journal of Sport Finance (IJSF), 7 (3), 359 - 379. Recuperado de http://web.a.ebscohost.com.bdatos. usantotomas.edu.co:2048/ehost/pdfviewer/pdfviewer? sid=73682080-d7d4 $-452 \mathrm{f}-9821-5 \mathrm{a} 9 \mathrm{~d} 7 \mathrm{a} 80 \mathrm{~d} 2 \mathrm{f} 6 \% 40$ sessionmgr4009\&vid=3\&hid=4206

Verdú-Pascual, C. L. (2008). Unidad didáctica: educación para la ciudadanía a través de los juegos y deportes alternativos. La Peonza. Revista de Educación Física para la paz, 3:87-9. 